\title{
Em Direção a um Modelo de Maturidade Tecnológica para Cidades Inteligentes
}

\author{
Kiev Gama ${ }^{1}$, Alexandre Alvaro ${ }^{2}$, Eduardo Peixoto ${ }^{1}$ \\ ${ }^{1}$ CESAR - Centro de Estudos e Sistemas Avançados do Recife \\ Rua Bione 220, Recife Antigo - Recife - PE - Brasil \\ ${ }^{2}$ Departamento de Computação (DComp) - Universidade Federal de São Carlos \\ (UFSCar) - Campus Sorocaba - Sorocaba - SP - Brasil \\ kiev.gama@cesar.org.br, alvaro@ufscar.br, eduardo.peixoto@cesar.org.br
}

\begin{abstract}
In order to deal with population growth, governments need to hire new policies for the creation of Smart Cities. Information and Communication Technologies (ICTs) are keys to achieve this goal. This article presents a discussion about the steps to be taken for gradually achieving smart cities. So, we propose a model of technological maturity.
\end{abstract}

Resumo. Para poderem lidar com o crescimento populacional desenfreado, governos necessitam empregar novos dispositivos estratégicos para a criação de Cidades Inteligentes. As Tecnologias de Informação e Comunicação (TICs) são fundamentais para alcançar este objetivo. Este artigo traz uma discussão a respeito das etapas a serem seguidas para gradualmente se alcançar um nível ideal de Cidade Inteligente. Para tal, propomos um modelo de maturidade tecnológica.

\section{Introdução}

O termo "cidades inteligentes" refere-se a um dispositivo estratégico para o planejamento e gestão inteligente de cidades. O conceito também é visto por alguns autores como algo associado à atração de capital humano [Glaeser e Berry 2006], ou mesmo a combinação deste aspecto com a qualidade de vida [Shapiro 2006] na tentativa de explicar o rápido crescimento de cidades que abrigam pessoas altamente capacitadas. Entretanto, o termo "cidades inteligentes" tem sido, cada vez mais, relacionado ao emprego eficiente de Tecnologias de Informação e Comunicação (TICs) como uma ferramenta para melhorar a infraestrutura e serviços da cidade, consequentemente trazendo melhor qualidade de vida.

Mahizhnan [Mahizhnan 1999] relata a estratégia governamental fortemente baseada em TICs para transformar Cingapura em uma cidade inteligente. Em [Odendaal 2003] verifica-se que TICs foram a estratégia em Brisbane (Austrália), e Durban (África do Sul), para melhorar aspectos de governança. No contexto de cidades inteligentes, diversas TICs podem ser utilizadas, tais como Internet das Coisas [Ahson 2008]; sensores diversos (ex: RFID e ZigBee); sistemas de informação; computação em nuvem para armazenar e aumentar a eficiência de aplicações; mobilidade com aplicações para smartphones e tablets; business intelligence como forma de minerar dados para apoio à decisão, dentre outros, tornando possível a coleta, processamento, distribuição e análise que facilitem a tomada de decisão estratégica governamental ou da população.

Por outro lado, é necessária a criação de sistemas capazes de lidar com toda a massa de informação criada, seja através de sistemas complexo para análise dos dados seja através de infraestrutura para interligar estes sistemas. Níveis de maturidade 
tecnológica serviriam como guia gradativo rumo à construção de uma cidade inteligente, auxiliando na tomada de decisão, gestão e planejamento estratégico das cidades.

Neste âmbito, este artigo apresenta um Modelo de Maturidade Tecnológica (MMT) para cidades inteligentes visando auxiliar no processo de amadurecimento gradativo das cidades em TICs e trazer para a comunidade acadêmica uma discussão sobre como deve-se empregar TICs no contexto de cidades inteligentes. Assim, a seção seguinte apresenta os trabalhos relacionados em áreas correlatas; a seção 3 apresenta conceitos relacionados aos domínios relevantes para cidades inteligentes; na seção 4 é apresentado o papel de TICs no contexto de cidades inteligentes; na seção 5 é descrito o modelo de maturidade tecnológica para cidades inteligentes; e por final a seção 6 apresenta conclusões e possibilidades de trabalhos futuros.

\section{Trabalhos Relacionados: Modelos de Maturidade em outros contextos}

O CMM (Capability Maturity Model) [Paulk 1993] é um modelo precursor referente à maturidade ligada a processos, servindo de referência para outros modelos como CMMI, MPS.Br, BPMN, dentre outros. A idéia de ter um modelo de maturidade serve como referencial para que as organizações evoluam continuamente seu processo de desenvolvimento de software e, consecutivamente, aumentem a qualidade do software produzido e obtenham uma maior aceitação do mercado.

Em outro contexto relacionado ao de cidades inteligentes, encontra-se o modelo de maturidade de redes inteligentes (Smart Grid Maturity Model - SGMM) [SEI 2011]. Este modelo é uma ferramenta gerencial que define cinco níveis (iniciado, habilitado, integrado, otimizado e pioneiro), com o objetivo de ajudar instituições a planejarem suas redes elétricas inteligentes e medirem o progresso em níveis de maturidade. O modelo foi planejado, testado e validado em casos reais pelo SEI e atualmente o SEI trabalha com consultoria para auxiliar empresas/cidades interessadas em avaliar seu nível de maturidade neste segmento.

Concomitantemente, este trabalho visa apresentar um modelo de maturidade tecnológica para cidades inteligentes baseado em domínios diversos que são interessantes para uma cidade se tornar mais automatizada, gradativamente. Cada domínio é avaliado sob três fatores e um conjunto de critérios para avaliar qual nível de maturidade a cidade possui naquele momento.

\section{Domínios alvo das Cidades Inteligentes}

Não há um consenso em relação aos eixos (aqui chamados de domínios) fundamentais de cidades inteligentes. Segundo um modelo europeu [Vienna 2007], o conceito de smart cities envolve seis domínios: Economia, Mobilidade, Governança, Meio-ambiente, Qualidade de vida e Capital Humano. Em [Dirks e Keeling 2010], a IBM apresenta como domínios os cidadãos, os serviços da cidade, negócios, transporte, comunicação, água e energia. Entretanto, em sua iniciativa de Smarter Cities $^{1}$ a empresa utiliza domínios diferentes daqueles, combinados com um conjunto de TICs ligadas a cidades inteligentes, resultando numa lista com mais de vinte conceitos.

O próprio Simpósio Brasileiro de Sistemas de Informação [SBSI 2012] enumera os seus domínios de interesse (transporte, educação, comunicação, saúde, água e segurança) que diferem daqueles outros, apesar das diversas interseções. De forma geral, não se pode enumerar uma lista restritiva determinando os domínios alvo de uma cidade

\footnotetext{
${ }^{1}$ http://www.ibm.com/smartercities/
} 
inteligente. A lista deve variar de acordo com o contexto e prioridades de cada cidade. Enquanto algumas cidades possuem o tráfego saturado e necessitam de soluções inteligentes para controlar o trânsito, outras podem não ter este domínio como ponto crítico e priorizar, por exemplo, aspectos de governança e saúde.

\section{Tecnologias de Informação e Comunicação em Cidades Inteligentes}

Exemplos de TICs como computação em nuvem, redes de sensores sem fio, redes elétricas inteligentes, sistemas de informação geográficos e dispositivos móveis já são utilizadas no contexto de cidades inteligentes e podem ser potencializados em soluções integradas e mais elaboradas. $\mathrm{O}$ uso de tecnologia pode vir desde a coleta de dados na rua até níveis mais altos, fornecendo mecanismos que auxiliem no monitoramento da cidade e na tomada de decisões.

Conforme ilustrado em [OECD 2010], redes de sensores sem fio já são utilizadas em diversos contextos ligados às cidades inteligentes, tais como transporte, prédios inteligentes, redes inteligentes e monitoramento ambiental. Este tipo de tecnologia permearia a cidade com diferentes tipos de sensores que coletariam dados a serem analisados para extrair-se informação num contexto mais amplo (ex: inundações, congestionamento no trânsito, padrões de consumo de eletricidade).

A computação em nuvem também vem sendo utilizada em domínios ligados a cidades inteligentes. Em [Simmhan et al 2010], o uso da nuvem no contexto de redes inteligentes é justificado pela escalabilidade e facilidade de integrar dados da rede provenientes de diversas origens, para posterior análise. No âmbito de governança, o projeto europeu EPIC [Ballon et al 2011] propõe uma plataforma baseada em nuvem computacional para fornecer diversos serviços aos cidadãos.

Sistemas de Informação Geográfica (SIG) são úteis no contexto de cidades inteligentes em domínios como transporte (visualização de rotas e dados de tráfego) e saúde (mapeamento de epidemias). TICs são fácilmente acessíveis (Web, dispositivos móveis), inserindo pessoas como participantes ativos (consumidores e produtores de dados e serviços) em uma cidade inteligente. As TICs atuais permitem que cidadãos atuem de forma participativa alimentando SIGs com "informações geográficas voluntariadas" [Goodchild 2007]. Exemplos utilizando tecnologia móvel são o Waze ${ }^{2}$ e o Wabbers ${ }^{3}$, aplicativos que permitem usuários de smartphones dotados de GPS mapearem a cidade e agregar informações geolocalizadas (buracos, trânsito, blitz, etc).

\section{Modelo de Maturidade Tecnológica (MMT)}

A diversidade tanto de domínios de problemas como de tecnologias a serem empregadas pode dificultar a criação de soluções aplicáveis a todos os domínios de uma cidade. Apesar de ser necessária uma visão holística do problema das cidades, defendemos a ideia de que o processo de tornar uma cidade numa cidade inteligente pode ser gradativo, e executado individualmente em cada domínio de problema, convergindo-os apenas nas etapas finais. Sendo assim, a motivação é usar o Modelo de Maturidade Tecnológica (MMT) como referencial no uso de TICs em soluções voltadas para cidades inteligentes. A intenção final é alcançar um nível otimizado no uso de recursos tecnológicos integrando as diferentes soluções empregadas em cada domínio.

\footnotetext{
2 http://www.waze.com/

3 http://www.wabbers.com/
} 
A concretização da visão de uma cidade inteligente inclui a integração de dispositivos (conceituais e físicos) variados. As ações envolvem a integração de diferentes sistemas de coleta de dados, que estarão interligados através da nuvem. Cada sistema pode empregar abordagens tecnológicas diferentes e possivelmente a integração com sistemas legados. $\mathrm{O}$ processo de implantação para alcançar o nível de eficiência ideal de uma cidade inteligente deve ser gradativo. O modelo gradual proposto neste trabalho está dividido em cinco níveis, conforme ilustrado na Figura 1.

0. Caótico: Fase onde a maioria das cidades inicia. Nesta fase as cidades não possuem TICs para auxiliar no processo de gerenciamento da cidade. Podem até possuir alguns dados das poucas informações que obtém de serviços básicos prestados a população porem não fazem uso destes dados. Ainda, a população pode utilizar aplicativos (ex: Waze e Wabbers, citados anteriormente) com dados em tempo real em seus smartphones, sem serem "endossados" por órgãos governamentais. Adicionalmente, as redes sociais fazem um papel extremamente importante nesta fase onde a população pode colaborar com informações sobre a cidade em tempo real.

1. Inicial. Fase de planejamento e modelagem de sistemas de informação que irão auxiliar em determinado domínio assim como a identificação de sistemas existentes que potencialmente podem ser integrados à solução da cidade inteligente. Neste nível podese existir alguma automação na captura dos dados a serem gerenciados, como uso de sensores diversos como RFID, ZigBee; introdução do conceito de Internet das Coisas [Ahson 2008] no desenvolvimento de aplicações, dentre outras opções.

2. Gerenciado. Dados coletados (i.e. dados de tráfego, dados de consumo de energia, qualidade de água, etc) e acessíveis através de sistemas de informação. Por exemplo, SIGs podem ser empregados neste nível para visualizar dados por região; dados podem servir para a geração de informação de alto valor agregado (ex: rotas de trânsito baseadas em dados em tempo real sobre o tráfego); sensores podem ser alojados no leito dos rios e represas visando informar a população via SMS quando uma possível enchente pode acontecer, dentre outras possibilidades.

3. Integrado. Cidade inteligente com sistemas utilizando o modelo de computação em nuvem, estando integrados e disponíveis na forma de serviços tanto para cidadãos como para aplicações de terceiros. O cidadão tem papel ativo como usuário dos serviços da nuvem como alimentador de dados, através de seus dispositivos móveis que se comunicam com a nuvem. Nesta fase o uso de computação ubíqua e autonômica são essências visando estar disponível em todo e qualquer lugar e visando reduzir necessidade de manutenção, respectivamente. O governo deveria funcionar como facilitador da cidade inteligente e fomentador de um ecossistema de serviços. Governo gerando oportunidades de negócios para empresas terceiras agregarem serviços.

4. Otimizado. Cidade eficiente, buscando inovar e ser pioneira nas soluções de TICs. Apoio à tomada de decisões utilizando os dados obtidos dos diversos domínios da cidade; uso de informações para sistemas de previsão de eventos, sejam climáticos ou não; suporte a tomada de decisão tanto para a população quanto para governantes, ou seja, tanto informação para o munícipe quanto informação para o governante tomar decisões. Neste nível entra em ação sistemas de apoio a decisão para auxiliar nas decisões estratégicas das cidades. 


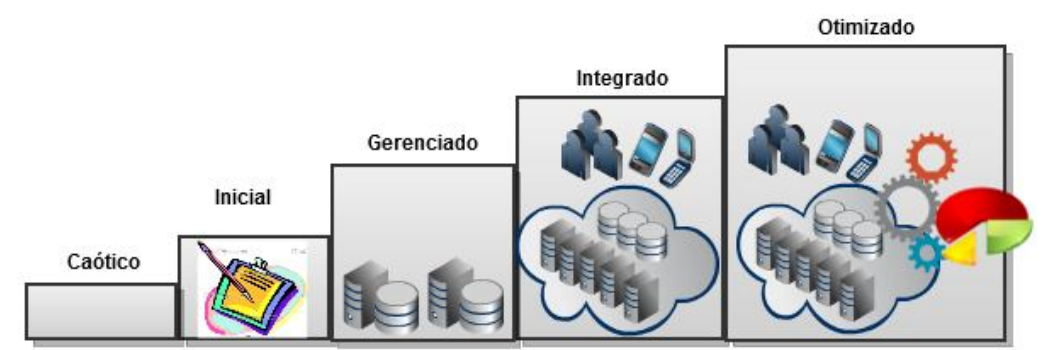

Figura 1. Níveis de maturidade tecnológica de cidades inteligentes

O nível de maturidade tecnológica no processo de otimização de cidades pode ser avaliado por domínio. É importante que a cidade tenha conhecimento de todos os domínios e respectivos critérios visando planejar a inserção de TICs da melhor forma possível. Assim, as cidades podem selecionar, em um primeiro momento e de forma isolada, o domínio mais importante no qual irão investir soluções empregando maturidade tecnológica. À medida que o nível de maturidade de cada domínio vai crescendo, se faz necessária a integração entre eles. A Tabela 1 apresenta alguns dos domínios que podem ser considerados no MTT, assim como exemplo de critérios adotados para avaliação de cada fase.

Tabela 1. Matriz de maturidade tecnológica aplicada por domínio

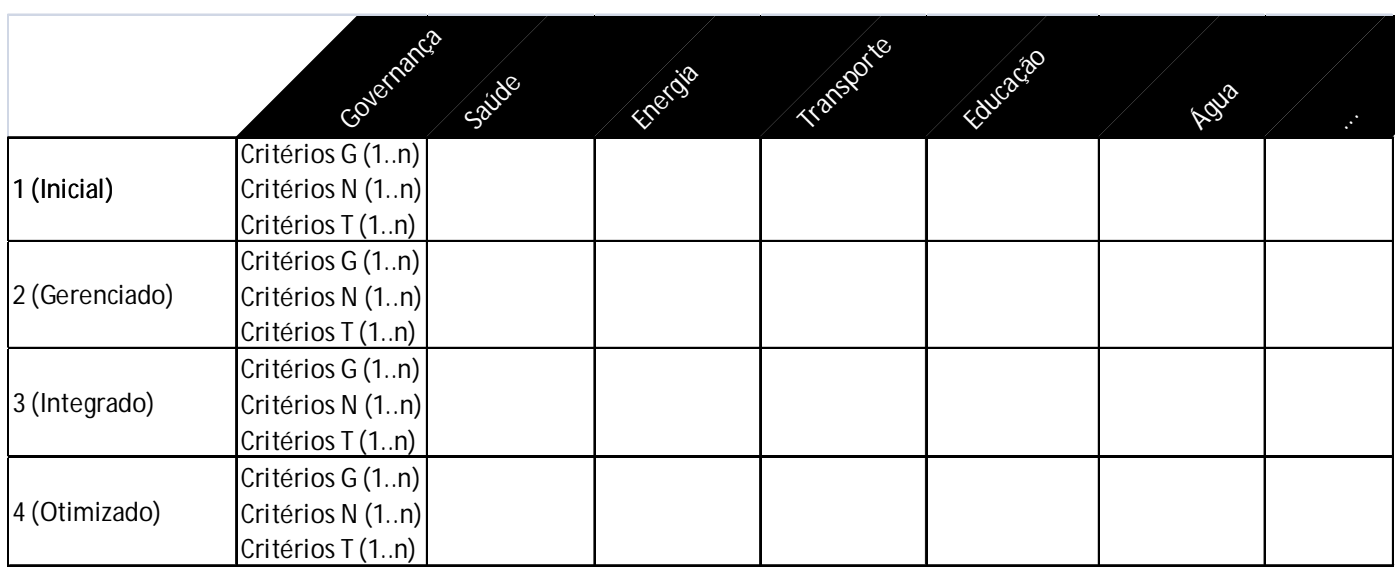

Como pode ser visto na Tabela 1, os critérios são baseados nos fatores: gente $(\mathrm{G})$, negócios (N) e tecnologia (T). Gente (engloba governo e cidadãos) é fator necessário, pois é o engajamento de pessoas que tornará efetivo o uso das soluções de TICs. O fator Negócios refere-se a gerar um modelo econômico sustentável acerca do uso da infraestrutura e serviços de TICs no contexto de cidades inteligentes. $\mathrm{O}$ fator Tecnologia está ligado à adequação de TICs aos cenários e necessidades das cidades. Cada fator deverá conter uma lista determinada de critérios (metas) que precisam ser alcançados para cumprir com os requisitos de cada nível do MMT. As práticas, assim como os critérios, ainda estão em fase de definição e serão baseados nos modelos correlatos de outras áreas como visto na seção 2 (por exemplo, a ideia de áreas de processo, metas e práticas que estão definidas no CMMI [SEI 2012]).

A população deve se envolver colaborativamente no fornecimento de informações dos diversos domínios bem como na avaliação das informações disponibilizadas. Com base nisso, a população terá influencia direta nos resultados das decisões estratégicas adotadas pelo governo. Paralelamente, o fator negócios se faz necessário criar um ecossistema de serviços baseado em TICs visando prover serviços diversos para alcançar meios de tornar a cidade inteligente. Por fim, o engajamento 
governamental é fator fundamental para garantir que os níveis de maturidade sejam alcançados. Este fator foi decisivo para o sucesso das estratégias empregadas em Cingapura [Mahizhnan 1999] e uma grande preocupação em Durban [Odendaal 2003].

\section{Conclusão e Trabalhos Futuros}

Colocar o conceito de cidades inteligentes em prática requer o uso de TICs como ferramenta auxiliar. Empregar TICs de maneira otimizada é fundamental para auxiliar no controle e eventual tomada de decisões em face a problemas comumente encontrados ao se por em prática estratégias para cidades inteligentes. Assim, este artigo apresentou um modelo de maturidade no uso de TICs em cidades inteligentes, e inclui fatores que podem influenciar a adoção de tecnologias assim como maximizar os resultados que elas podem trazer. Como trabalhos futuros serão investigados e validados os critérios de cada nível e domínio do MMT, que poderiam ser validados em estudos em cidades como Recife/PE e Sorocaba/SP visando obter feedback do modelo proposto. Ainda, serão desenvolvidas ferramentas para coleta de informações das cidades.

\section{Referências}

Ahson, S. and Ilyas, M. (2008) "RFID HANDBOOK: Application, Technology, Security and Privacy”. In: CRC Press, Boca Raton, FL.

Ballon, P., Glidden, J., Kranas, P., Menychtas, A., Ruston, S., Van der Graa, S. (2011) "Is there a Need for a Cloud Platform for European Smart Cities?". eChallenges e-2011 Conference Proceedings.

Dirks, S., Keeling, M. (2010) “A vision of smarter cities: How cities can lead the way into a prosperous and sustainable future". IBM Inst. for Business Value, Exec. Report.

Glaeser, E.L., Berry, C. R. (2006). "Why are smart places getting smarter?", Taubman Center Policy Brief, Harvard Kennedy School, March 2006.

Goodchild, M. F. (2007). Citizens as sensors: the world of volunteered geography. GeoJournal 69, p. 211221.

Mahizhnan, A. (1999). "Smart Cities: the Singapore Case". Cities, Volume 16, Issue 1, p. 13-18.

OECD (2010), OECD Information Technology Outlook 2010, OECD Publishing.

Odendaal, N. (2003) "Information and communication technology and local governance: understanding the difference between cities in developed and emerging economies", Computers, Environment and Urban Systems, vol. 27, issue 6, Nov. 2003, p. 585-607.

Paulk, M.C., Curtis, B., Chrissis, M.B., Weber, C.V. (1993) "Capability Maturity Model for Software, Version 1.1" CMU/SEI-93-TR-24, SEI, Pittsburgh, PA.

Shapiro, J.M. (2006) "Smart Cities: Quality of Life, Productivity, and the Growth Effects of Human Capital", In: The Review of Economics and Statistics, vol. 88, n. 2, p. 324-335.

Simmhan, Y., Giakkoupis, M., Cao, B., Prasanna, V. K. (2010) "On Using Cloud Platforms in a Software Architecture for Smart Energy Grids,” IEEE International Conference on Cloud Computing.

Software Engineering Institute, SEI (2011). "Smart Grid Maturity Model Update”, vol. 3. Disponível em http://www.sei.cmu.edu/library/assets/brochures/SGMM_2011.pdf Acesso em 05/01/2012

Software Engineering Institute, SEI (2012). Capability Maturity Model Integration. Disponível em: http://www.sei.cmu.edu/cmmi/ Acesso em 05/01/2012

VII Simpósio Brasileiro de Sistemas de Informação, SBSI (2012). Disponível em: http:// www.each.usp.br/sbsi2012 Acesso em 05/01/2012

Vienna University of Technology, Centre of Regional Science (2007). "Smart cities Ranking of European medium-sized cities", final report. Disponível em: http://www.smartcities.eu/download/smart_cities_final_report.pdf Acesso em 05/01/2012 